\title{
Predictive Factors of Severe Toxicities of Pemetrexed-Containing Chemotherapy in Patients with Non-Squamous Non-Small Cell Lung Cancer
}

\author{
Yuki Katsuya, Yuichiro Takeda*, Go Naka, Haruhito Sugiyama \\ Department of Respiratory Medicine, National Center for Global Health and Medicine, Tokyo, Japan \\ Email: *ytakeda@hosp.ncgm.go.jp
}

How to cite this paper: Katsuya, Y., Takeda, Y., Naka, G. and Sugiyama, H. (2017) Predictive Factors of Severe Toxicities of Pemetrexed-Containing Chemotherapy in Patients with Non-Squamous Non-Small Cell Lung Cancer. Journal of Cancer Therapy, 8, 1030-1039.

https://doi.org/10.4236/jct.2017.811087

Received: October 27, 2017

Accepted: November 25, 2017

Published: November 28, 2017

Copyright $\odot 2017$ by authors and Scientific Research Publishing Inc. This work is licensed under the Creative Commons Attribution International License (CC BY 4.0).

http://creativecommons.org/licenses/by/4.0/

\section{(c) (i) Open Access}

\begin{abstract}
Background: Pemetrexed (PEM) is an efficacious multi-targeted antifolate with acceptable toxicities for non-squamous non-small cell lung cancer (nonSq NSCLC). However, in the clinical setting, PEM has more severe adverse effects than those reported. The aim of this study was to identify the factors for the toxicities of PEM-containing chemotherapy in non-Sq NSCLC patients in the clinical setting. Patients and Methods: We retrospectively evaluated the factors related to PEM toxicities in chemotherapy-naive patients with non-Sq NSCLC from September 2009 to July 2013 at our hospital. Logistic regression model was used in the univariate and multivariate analyses. Results: In total, 104 patients were analyzed. Grades 3 to 5 hematologic toxicities were frequent and included neutropenia (42\%), febrile neutropenia (7\%), anemia (18\%), thrombocytopenia (17\%), and disseminated intravascular coagulation (2\%). On multivariate analyses, the predictors were poor performance status (PS) [odds ratio (OR): 4.89, 95\% confidence interval (CI): 1.22 - 19.4] and low body mass index (OR: 1.44, 95\% CI: 1.05 - 1.98) for febrile neutropenia; concomitant chronic infectious disease (OR: 6.63, 95\% CI: 1.59 - 27.5) and bevacizumab use (OR: 3.57, 95\% CI: 1.36 - 9.32) for neutropenia; poor PS (OR: $3.02,95 \%$ CI: 1.33 - 6.85) for thrombocytopenia; and low serum albumin level (OR: 0.22, 95\% CI: 0.08 - 0.63) for non-hematologic toxicities. Conclusions: In addition to the previously reported predictors of PEM toxicities, the presence of concomitant chronic infectious disease was associated with hematologic toxicities. Patient groups who are not sufficiently evaluated in clinical trials should be carefully monitored for the development of more toxicities than expected.
\end{abstract}




\section{Keywords}

Non-Squamous Non-Small Cell Lung Cancer, Pemetrexed, Toxicity, Clinical Settings

\section{Introduction}

Lung cancer is the major cause of cancer-related deaths worldwide [1] [2]. Current guidelines recommend that newly diagnosed or chemotherapy-naive patients with advanced non-squamous non-small cell lung cancer (non-Sq NSCLC) who have good Eastern Cooperative Oncology Group Performance Status (PS) receive 4 to 6 cycles of platinum-based chemotherapy. Pemetrexed (PEM), which is an inhibitor of thymidylate synthase and other folate-dependent enzymes, including dihydrofolate reductase and glycinamide ribonucleotide formyl transferase, is one of the key effective drugs with mild toxicities for the treatment of non-Sq NSCLC [3]. Serum homocysteine and methylmalonic acid levels were identified as important predictive factors for severe toxicity caused by PEM. At present, patients are given vitamin B12 and folic acid supplementation before administration of PEM-containing chemotherapy [4]; despite this PEM has been reported by some to induce severe hematologic toxicities [5], rhabdomyolysis [6], and renal failure [7]. To date, age, estimated glomerular filtration rate (eGFR), renal function, vitamin supplementation, ethnic origin, and regular use of nonsteroidal anti-inflammatory drugs have been the reported predictors of PEMrelated hematologic or non-hematologic toxicities [5] [8] [9] [10] [11]. The aim of this study was to explore the clinical and biochemical factors related to the toxicities of PEM-containing chemotherapy in patients with non-Sq NSCLC in the clinical setting.

\section{Patients and Methods}

\subsection{Study Design and Patients}

We analyzed patients with advanced non-Sq NSCLC who visited the Department of Respiratory Medicine of National Center for Global Health and Medicine between September 2009 and July 2013. Patients were excluded if they were not treated with PEM-containing chemotherapy as a first line regimen, if they received concurrent radiotherapy, or if chemotherapy was given as an adjuvant modality.

We retrospectively reviewed the medical records for hematologic and other clinical data from the date of treatment initiation to 28 days after the last administration of PEM. Hematologic and non-hematologic toxicities were graded according to the National Cancer Institute Common Terminology Criteria for Adverse Events version 4.0. This retrospective analysis was completed in December 2013, during a time when ethics committee approval was not necessary for retrospective analyses in Japan. However, the patients themselves gave in- 
formed consent before receiving PEM-containing chemotherapy.

\subsection{Statistical Analyses}

To determine the predictors of adverse events, univariate and multivariate logistic regression analyses were performed. In the univariate analysis, the following 16 variables were included as explanatory variables: age, sex, PS, stage, smoking status, epidermal growth factor receptor (EGFR) mutation status, concurrent use of platinum (cisplatin or carboplatin), concurrent use of bevacizumab, renal function (eGFR or creatinine clearance), serum albumin level, lymphocyte count, concomitant chronic infectious disease, coexisting cancer, pretreatment with EGFR-tyrosine kinase inhibitor, and body mass index (BMI). Spearman's rank test was used to assess the correlation between the independent variables and each toxicity; a correlation coefficient of $>0.3$ indicated an association. For the selection of variables for multivariate analysis, a stepwise method was used with significance level set at a p-value of $<0.15$. In the multivariate analysis, statistical significance was determined with a two-sided test at a p-value of $<0.05$. All analyses were performed using SPSS Statistics software version 19 (IBM, New York, USA).

\section{Results}

\subsection{Patients}

Among 275 consecutive patients who visited our department, 104 patients with chemotherapy-naive advanced non-SQ NSCLC were treated with PEM-containing chemotherapy (Figure 1); their characteristics are shown in Table 1. Median age was 67 years, men comprised 54\%, 87\% were in stage IV, and $90 \%$ had a PS of 0 to 1 . EGFR mutation was positive in $38 \%$; never-smokers comprised $34 \%$, whereas heavy smokers, defined as those who had a smoking index of more than 600 , comprised $58 \%$. Platinum doublet regimen was used in $87 \%$, whereas bevacizumab was used in $28 \%$. The BMI of $23 \%$ patients was lower than 18.5 .

\subsection{Chemotherapy-Related Toxicity}

For grade 3 to 5 hematologic toxicities, neutropenia was observed in $42 \%$, febrile

Advancer non-squamous non-small cell lung cancer from September 2009 through July 2013, $N=275$

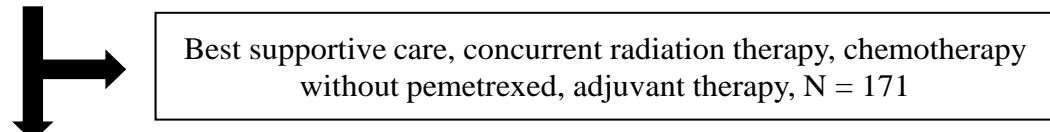

Chemotherapy-naive patients treated with pemetrexed-containing chemotherapy, $\mathrm{N}=104$

Figure 1. Study flow chart. 
Table 1. Patient characteristics $(\mathrm{N}=104)$.

\begin{tabular}{|c|c|c|c|}
\hline & Variable & $\mathrm{N}$ & $\%$ \\
\hline \multicolumn{4}{|l|}{ Gender } \\
\hline & Male & 57 & 54 \\
\hline & Female & 47 & 46 \\
\hline Age years (median, range) & & 67 & $38-85$ \\
\hline \multicolumn{4}{|l|}{ Stage } \\
\hline & Relapse & 5 & 5 \\
\hline & IIIA/B & 8 & 8 \\
\hline & IV & 91 & 87 \\
\hline \multicolumn{4}{|l|}{ EGFR mutation } \\
\hline & Positive & 39 & 38 \\
\hline & Negative & 60 & 58 \\
\hline & Unknown & 5 & 4 \\
\hline \multicolumn{4}{|l|}{ Smoking history } \\
\hline & $\mathrm{SI} \geq 600$ & 60 & 58 \\
\hline & $0<\mathrm{SI}<600$ & 9 & 8 \\
\hline & $\mathrm{SI}=0$ & 35 & 34 \\
\hline \multicolumn{4}{|l|}{ ECOG PS } \\
\hline & 0 & 32 & 31 \\
\hline & 1 & 61 & 59 \\
\hline & 2 & 9 & 8 \\
\hline & 3 & 2 & 2 \\
\hline BMI (median, range) & & 21.3 & $13.7-30.4$ \\
\hline \multicolumn{4}{|l|}{ Chemotherapy regimen } \\
\hline & CBDCA + PEM & 40 & 38 \\
\hline & $\mathrm{CBDCA}+\mathrm{PEM}+\mathrm{Bev}$ & 21 & 20 \\
\hline & CDDP + PEM & 23 & 22 \\
\hline & $\mathrm{CDDP}+\mathrm{PEM}+\mathrm{Bev}$ & 4 & 4 \\
\hline & PEM & 12 & 12 \\
\hline & $\mathrm{PEM}+\mathrm{Bev}$ & 4 & 4 \\
\hline
\end{tabular}

EGFR $=$ epidermal growth factor receptor, $\mathrm{SI}=$ smoking index, ECOG $=$ Eastern Cooperative Oncology Group, $\mathrm{PS}=$ performance status, $\mathrm{BMI}=$ body mass index, $\mathrm{PEM}=$ pemetrexed, $\mathrm{CBDCA}=$ carboplatin, $\mathrm{Bev}=$ bevacizumab, CDDP = cisplatin.

neutropenia in $7 \%$, anemia in $18 \%$, thrombocytopenia in $17 \%$, and disseminated intravascular coagulation in $2 \%$. For grade 3 to 4 non-hematologic toxicities, anorexia was seen in $4 \%$, nausea in $3 \%$, rash in $3 \%$, drug-induced pneumonitis in $2 \%$, thromboembolic event in $2 \%$, hyponatremia in $1 \%$, hemoptysis in $1 \%$, creatinine elevation in $1 \%$.

\subsection{Risk Factors for Toxicities}

Table 2 shows the risk factors for hematologic toxicities. After excluding each 


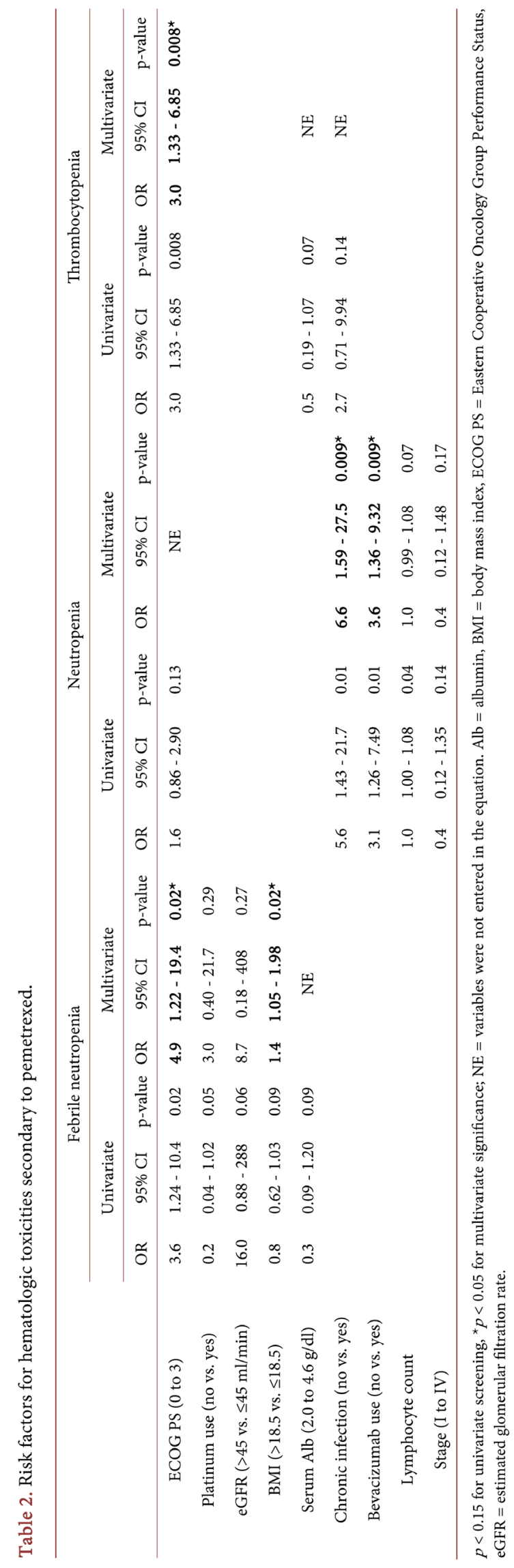


correlated variable by the Spearman's rank test, the best model for febrile neutropenia was determined to include PS, BMI, serum albumin, eGFR, and use of platinum. In the multivariate analysis using this model, the predictors for febrile neutropenia were PS [odds ratio (OR): 4.9, 95\% confidence interval (CI): 1.22 19.4, $p=0.02$ ] and BMI (OR: 1.4, 95\% CI: 1.05 - 1.98, $p=0.02$ ); those for neutropenia were concomitant chronic infectious disease (OR: 6.6, 95\% CI: 1.59 27.5) and use of bevacizumab (OR: 3.6, 95\% CI: 1.36 - 9.32); and that for thrombocytopenia was PS (OR: 3.0, 95\% CI: $1.33-6.85, p=0.008$ ). For anemia, serum albumin level was the only significant risk factor (OR: 0.43, 95\% CI: 0.19 $0.99, p=0.047$ ) by univariate logistic analysis. On multivariate analysis, serum albumin was a predictor of non-hematologic toxicities (OR: 0.22, 95\% CI: 0.08 $0.63, p=0.005$ ) (Table 3 ).

Twelve patients had concomitant chronic infectious disease (Table 4) secondary to hepatitis type $B$ virus $(n=3)$, hepatitis type $C$ virus $(n=2)$, human immunodeficiency virus $(\mathrm{n}=1)$, latent Mycobacterium tuberculosis $(\mathrm{n}=1)$, and non-tuberculous mycobacteria $(n=7)$. Of these patients, 10 experienced grade 3 to 4 hematologic toxicities, including febrile neutropenia in $17 \%$, neutropenia in $83 \%$, anemia in $8 \%$, and thrombocytopenia in $33 \%$.

\section{Discussion}

Poor PS, low BMI, concomitant chronic infectious disease, use of bevacizumab, and low serum albumin level were related to PEM-induced toxicities in patients with non-Sq NSCLC. The results were consistent with those of previous reports; use of bevacizumab was said to increase the risk for neutropenia and febrile neutropenia and poor PS was a risk for treatment-related deaths [12] [13]. Low BMI or muscularity was reported to be associated with high incidence of toxicities during chemotherapy for renal cell carcinoma that has undergone sorafenib

Table 3. Risk factors for non-hematologic toxicities secondary to pemetrexed.

\begin{tabular}{ccccccc}
\hline & \multicolumn{5}{c}{ Non-hematologic toxicities } \\
\cline { 2 - 6 } & \multicolumn{5}{c}{ Univariate } & \multicolumn{3}{c}{ Multivariate } \\
\cline { 2 - 6 } & OR & $95 \%$ CI & p-value & OR & $95 \%$ CI & p-value \\
\hline $\begin{array}{c}\text { Serum Alb } \\
(2.0 \text { to } 4.6 \text { g/dl) }\end{array}$ & 0.2 & $0.08-0.58$ & 0.002 & 0.2 & $0.08-0.63$ & $0.005^{*}$ \\
Age & 1.1 & $1.00-1.13$ & 0.05 & 1.1 & $1.00-1.15$ & 0.05 \\
$\begin{array}{c}\text { Ccr }(>45 \text { vs. } \leq 45 \\
\text { ml/min })\end{array}$ & 1.0 & $0.93-1.00$ & 0.06 & & NE & \\
$\begin{array}{c}\text { Lymphocyte count } \\
\text { Platinum use } \\
(\text { no vs. yes })\end{array}$ & 1.0 & $0.89-1.00$ & 0.09 & & NE & \\
BMI & 0.4 & $0.10-1.17$ & 0.09 & & NE & \\
$(>18.5$ vs. $\leq 18.5)$ & 0.9 & $0.73-1.03$ & 0.11 & 0.9 & $0.74-1.07$ & 0.23 \\
\hline
\end{tabular}

$p<0.15$ for univariate screening, ${ }^{*} p<0.05$ for multivariate significance, $\mathrm{NE}=$ variables were not entered in the equation. $\mathrm{Alb}=$ albumin, $\mathrm{BMI}=$ body mass index, $\mathrm{Ccr}=$ creatinine clearance. 
Table 4. List of patients with concomitant chronic infectious diseases.

\begin{tabular}{cccc}
\hline Patient & Infectious disease & Current treatment & Toxicities \\
\hline 1 & Hepatitis type B virus & Entecavir & G3 neutropenia \\
2 & Hepatitis type B virus & Entecavir & - \\
& Hepatitis type B virus & & G4 neutropenia, G3 \\
thrombocytopenia
\end{tabular}

G, grade according to the National Cancer Institute Common Terminology Criteria for Adverse Events version 4.0.

treatment [14] and for metastatic breast cancer that has undergone capecitabine treatment [15]. Because $81 \%$ of PEM is bound to serum protein, the increased number of toxicities caused by hypoalbuminemia could be explained by the unbound PEM fraction in serum [16].

We showed that majority of patients with concomitant chronic infectious disease were at risk for hematologic toxicities. Patients treated with PEM-containing chemotherapy were routinely given both folic acid (1000 $\mathrm{mg} /$ day) and vitamin B12 (1000 mg intramuscularly every 9 weeks) [4]. In addition, vitamin B6 supplementation was reported to decrease the incidence of serious drug-related toxicities, such as neutropenia, diarrhea, mucosal toxicity, and infection [4] [16], by normalizing serum levels of homocysteine and cystathionine, which are markers for deficiency of folic acid and/or vitamin B12 and vitamin B6, respectively. Iso- 
niazid (INH) causes deficiency in biologically active vitamin B6 by interfering with its metabolism; therefore, patients treated with INH are routinely given pyridoxine for polyneuropathy prophylaxis [17]. In this study, all 4 patients treated with INH experienced relatively severe hematologic toxicities, which might have been caused by insufficient vitamin B6 level. Most of the published clinical trials on patients with cancer excluded those with concomitant chronic infectious diseases; as a result, the toxicities of PEM may have been underestimated.

This study had some limitations. One was its retrospective design. In addition, we did not measure serum cystathionine, homocysteine, and vitamin B6 and B12 levels. In order to evaluate the need for vitamin supplementation in a specific patient population, we are currently doing a prospective study that measures serum markers.

\section{Conclusion}

This study that was performed in the clinical setting showed that, in addition to previously-reported predictors of adverse effects of PEM treatment, such as low BMI, poor PS, use of bevacizumab, and hypoalbuminemia, the presence of concomitant chronic infectious disease was also associated with hematologic toxicities. In the clinical setting, patient populations that have not been sufficiently evaluated in clinical trials should be carefully monitored for more toxicities than expected.

\section{Funding}

This research did not receive any specific grant from funding agencies in the public, commercial, or not-for-profit sectors.

\section{Conflict of Interest}

No author has any conflict of interest related to this manuscript.

\section{References}

[1] Ferlay, J., Shin, H.R., Bray, F., Forman, D., Mathers, C. and Parkin, D.M. (2010) Estimates of Worldwide Burden of Cancer in 2008: GLOBOCAN 2008. International Journal of Cancer, 127, 2893-2917. https://doi.org/10.1002/ijc.25516

[2] Danaei, G., Vander Hoorn, S., Lopez, A.D., Murray, C.J. and Ezzati, M., (Cancers) CRAcg (2005) Causes of Cancer in the World: Comparative Risk Assessment of Nine Behavioural and Environmental Risk Factors. Lancet, 366, 1784-1793. https://doi.org/10.1016/S0140-6736(05)67725-2

[3] Scagliotti, G.V., Parikh, P., von Pawel, J., Biesma, B., Vansteenkiste, J., Manegold, C., Serwatowski, P., Gatzemeier, U., Digumarti, R., Zukin, M., Lee, J.S., Mellemgaard, A., Park, K., Patil, S., Rolski, J., Goksel, T., de Marinis, F., Simms, L., Sugarman, K.P. and Gandara, D. (2008) Phase III Study Comparing Cisplatin plus Gemcitabine with Cisplatin plus Pemetrexed in Chemotherapy-Naive Patients with Advanced-Stage Non-Small-Cell Lung Cancer. Journal of Clinical Oncology, 26, 3543 3551. https://doi.org/10.1200/JCO.2007.15.0375

[4] Niyikiza, C., Baker, S.D., Seitz, D.E., Walling, J.M., Nelson, K., Rusthoven, J.J., 
Stabler, S.P., Paoletti, P., Calvert, A.H. and Allen, R.H. (2002) Homocysteine and Methylmalonic Acid: Markers to Predict and Avoid Toxicity from Pemetrexed Therapy. Molecular Cancer Therapeutics, 1, 545-552.

[5] Kawazoe, H., Yano, A., Ishida, Y., Takechi, K., Katayama, H., Ito, R., Yakushijin, Y., Moriguchi, T., Tanaka, M., Tanaka, A. and Araki, H. (2017) Non-Steroidal Anti-Inflammatory Drugs Induce Severe Hematologic Toxicities in Lung Cancer Patients Receiving Pemetrexed plus Carboplatin: A Retrospective Cohort Study. PLoS One, 12, e0171066. https://doi.org/10.1371/journal.pone.0171066

[6] Huang, M.S., Tsai, J.R., Shen, M.C., Chou, S.H. and Yang, C.J. (2012) Pemetrexed as a Possible Cause of Severe Rhabdomyolysis in the Treatment of Lung Cancer. Lung Cancer, 76, 491-492. https://doi.org/10.1016/j.lungcan.2012.02.009

[7] Vootukuru, V., Liew, Y.P. and Nally Jr., J.V. (2006) Pemetrexed-Induced Acute Renal Failure, Nephrogenic Diabetes Insipidus, and Renal Tubular Acidosis in a Patient with Non-Small Cell Lung Cancer. Medical Oncology, 23, 419-422.

https://doi.org/10.1385/MO:23:3:419

[8] Kulkarni, P.M., Chen, R., Anand, T., Monberg, M.J. and Obasaju, C.K. (2008) Efficacy and Safety of Pemetrexed in Elderly Cancer Patients: Results of an Integrated Analysis. Critical Reviews in Oncology/Hematology, 67, 64-70.

https://doi.org/10.1016/j.critrevonc.2008.01.011

[9] Mita, A.C., Sweeney, C.J., Baker, S.D., Goetz, A., Hammond, L.A., Patnaik, A., Tolcher, A.W., Villalona-Calero, M., Sandler, A., Chaudhuri, T., Molpus, K., Latz, J.E., Simms, L., Chaudhary, A.K., Johnson, R.D., Rowinsky, E.K. and Takimoto, C.H. (2006) Phase I and Pharmacokinetic Study of Pemetrexed Administered Every 3 Weeks to Advanced Cancer Patients with Normal and Impaired Renal Function. Journal of Clinical Oncology, 24, 552-562. https://doi.org/10.1200/JCO.2004.00.9720

[10] Takimoto, C.H., Hammond-Thelin, L.A., Latz, J.E., Forero, L., Beeram, M., Forouzesh, B., de Bono, J., Tolcher, A.W., Patnaik, A., Monroe, P., Wood, L., Schneck, K.B., Clark, R. and Rowinsky, E.K. (2007) Phase I and Pharmacokinetic Study of Pemetrexed with High-Dose Folic Acid Supplementation or Multivitamin Supplementation in Patients with Locally Advanced or Metastatic Cancer. Clinical Cancer Research, 13, 2675-2683. https://doi.org/10.1158/1078-0432.CCR-06-2393

[11] Lara, P.N., Higdon, R., Lim, N., Kwan, K., Tanaka, M., Lau, D.H.M., Wun, T., Welborn, J., Meyers, F.J., Christensen, S., O’Donnell, R., Richman, C., Scudder, S.A., Tuscano, J., Gandara, D.R. and Lam, K.S. (2001) Prospective Evaluation of Cancer Clinical Trial Accrual Patterns: Identifying Potential Barriers to Enrollment. Journal of Clinical Oncology, 19, 1728-1733. https://doi.org/10.1200/JCO.2001.19.6.1728

[12] Soria, J.C., Mauguen, A., Reck, M., Reck, M., Sandler, A.B., Saijo, N., Johnson, D.H., Burcoveanu, D., Fukuoka, M., Besse, B., Pignon, J.P., Meta-Analysis of Bevacizumab in Advanced NSCLC Collaborative Group (2013) Systematic Review and MetaAnalysis of Randomised, Phase II/III Trials Adding Bevacizumab to PlatinumBased Chemotherapy as First-Line Treatment in Patients with Advanced NonSmall-Cell Lung Cancer. Annals of Oncology, 24, 20-30. https://doi.org/10.1093/annonc/mds590

[13] Ruckdeschel, J.C., Finkelstein, D.M., Ettinger, D.S., Creech, R.H., Mason, B.A., Joss, R.A. and Vogl, S. (1986) A Randomized Trial of the Four Most Active Regimens for Metastatic Non-Small-Cell Lung Cancer. Journal of Clinical Oncology, 4, 14-22. https://doi.org/10.1200/JCO.1986.4.1.14

[14] Antoun, S., Baracos, V.E., Birdsell, L., Escudier, B. and Sawyer, M.B. (2010) Low Body Mass Index and Sarcopenia Associated with Dose-Limiting Toxicity of Sora- 
fenib in Patients with Renal Cell Carcinoma. Annals of Oncology, 21, 1594-1598. https://doi.org/10.1093/annonc/mdp605

[15] Prado, C.M., Baracos, V.E., McCargar, L.J., Reiman, T., Mourtzakis, M., Tonkin, K., Mackey, J.R., Koski, S., Pituskin, E. and Sawyer, M.B. (2009) Sarcopenia as a Determinant of Chemotherapy Toxicity and Time to Tumor Progression in Metastatic Breast Cancer Patients Receiving Capecitabine Treatment. Clinical Cancer Research, 15, 2920-2926. https://doi.org/10.1158/1078-0432.CCR-08-2242

[16] Latz, J.E., Karlsson, M.O., Rusthoven, J.J., Ghosh, A. and Johnson, R.D. (2006) A Semimechanistic-Physiologic Population Pharmacokinetic/Pharmacodynamic Model for Neutropenia Following Pemetrexed Therapy. Cancer Chemotherapy and Pharmacology, 57, 412-426. https://doi.org/10.1007/s00280-005-0077-5

[17] van der Watt, J.J., Harrison, T.B., Benatar, M. and Heckmann, J.M. (2011) Polyneuropathy, Anti-Tuberculosis Treatment and the Role of Pyridoxine in the HIV/ AIDS Era: A Systematic Review. International Journal of Tuberculosis and Lung Disease, 15, 722-728. 